\title{
A Study on Using a Smartphone to Broadcast Basketball Games: Prototype Tests Based on a Video Calling/Conferencing Platform
}

\author{
K. L. Wang and Y. H. Chen
}

\begin{abstract}
Traditional mass media show less interest in primary student athletes games. As a result, those interested have little chance to watch the live broadcast of such a game. To service these people, the authors formed a six-person team that used Google Hangouts, a video calling/conferencing platform, to live stream games played in Level B games in the Taiwan's high-school basketball league (HBL) in 2016. The viewers who watched the live broadcasts completed post-game questionnaires. The survey for the first live broadcast contained open-ended questions that required the correspondents to give three advantages and three disadvantages of the live stream. A total of 28 questionnaires were then collected, encoded and analyzed statistically. The results showed that the top advantage is: 'attractive reporting style,' and the top disadvantage is: 'image quality not clear enough.' As for the second live broadcast, the research team asked close-ended questions. A total of 21 questionnaires showed that: (1) Having a sportscaster reporting the game attracted more spectators; (2) Poor lighting and shaky camera movements undermined the broadcasting quality; (3) Viewers/spectators would find a game more engaging if it is played by any of their friends; and (4) 'Real-time' broadcasting is the core value of user experience.
\end{abstract}

Index Terms-Video calling/conferencing platform, Google hangouts, high-school basketball league (HBL), live broadcast.

\section{INTRODUCTION}

The sports world interacts with the mass media, whose ubiquity has made sports a part of society [1]. The mass media is very important for the sports community to gain popularity, exposure and even sponsorships for the development of sports. While many people use the mass media to obtain information, sports fans rely heavily on it for pleasure [2], [3]. However, what we see on TV are often games in high-level professional leagues or tournaments such as the National Basketball Association (NBA), Major League Baseball (MLB) and Australia Open (AO). TV viewers have little chance to watch amateur sports, except for major events (e.g. the Olympic Games and the Asian Games). As for games played by student-athletes, TV networks tend to broadcast those at top level, such as the Division I games in the National Collegiate Athletic Association (NCAA) and the Level A games in Taiwan's High School Basketball League (HBL). It goes without saying that this is because such games

Manuscript received January 15, 2017; revised March 25, 2017

K. L. Wang is with the National Taiwan Sport University, Taoyuan City, Taiwan 33301 ROC (e-mail: elverz@ ms8.hinet.net).

Y. H. Chen was with National Taiwan Sport University, Taoyuan City, Taiwan 33301 ROC. She is now with the Department of Sports, Taipei City Government, Taiwan 10553 ROC (e-mail: good19930630@gmail.com). are more exciting and interesting. Another reason lies in broadcasting equipment, which can be extremely expensive. For example, satellite news gathering (SNG) devices usually cost over NT $\$ 100,000$ dollars (USD $\$ 3,200$ ). This causes TV networks to exclude games with low ratings from broadcasting lists. As a result, they tend to choose programs in the interests of their advertisers in order to gain more sponsorships.

All these factors account for why many games played by student-athletes at primary level are not televised. Sometimes even games at top level may not be broadcast for certain reasons. One example is the Group A games in the HBL played by female student-athletes. These games are not live broadcast due to limitations imposed by hardware or facilities [4]. However, there still is the demand for broadcasting such a game because the friends, families and opponents of the players care about it. So it makes every sense to live broadcast untelevised games as a way to fill the unmet commercial demand and to gain exposure for every player.

The recent popularity of smartphones and the Internet's increasing stability facilitate the broadcasting of daily activities, which now costs very little. Since 2016, when Facebook offered a live streaming service, there have been more and more games at various levels broadcast on the platform. Smartphones and other similar gadgets serve as a handy solution to the issue discussed above. However, there is a difference between broadcasting of sports games and that of daily life. Sports games, for their part, need to be watched from various camera angles and anchored by at least an anchor, sometimes even with a commentator giving professional comments. Put simply, sports broadcasting (i.e. sportscasting) calls for teamwork. The purpose of this research is to explore whether it is possible to simply use a smartphone to live broadcast sports games and what might be a problem. We thus devised the idea of using a video calling/conferencing program to do so.

To this end, we designed a prototype model to address the aforementioned issues. We hope that the results of our research can serve as a reference for hardware developers, media companies and even social networking platform managers. The ultimate purpose is to discover a good tool or model so that more athletes at primary level can be seen.

\section{LITERATURE REVIEW}

\section{A. Online Live Streaming $(O L S)$}

The term 'streaming' refers to data transmission on the Internet. In many cases, it actually means multimedia 
streaming. Before opening or viewing, the downloader of a file used to wait until it is completely received. In literature, this process is termed as 'downloadable media' [5] and might not work well for bulk data transfer as the downloader had to wait for a long time and there had to be large space for data storage on the receiving end. To tackle this problem, the information industry developed a solution that allows for simultaneous playback of already-downloaded sections of data. Called 'streaming media,' this feature works wonders when users are processing massive multimedia data [6]. Streaming media, in its early stage, was mostly applied to video-on-demand $(\mathrm{VoD})$ models, so some researchers also refer to it as 'storing and forwarding video' in some literature [7].

The technology of streaming media has been widely applied since its introduction. RealAudio 1.0, the earliest application of streaming media, was introduced in March 1995. It transmitted data between a single server and a single player. Then a cooperative networking model was used for transmission, with the content format varying from pre-recorded files to live playback [8], [9]. Our prototype model processes live streams, so we call it 'online live streaming (OLS).' Viewers of live streams need to use Internet-enabled devices. The model also derived the name 'live stream broadcasting (OSB)' as it is similar to satellite-based live broadcasting by nature.

\section{B. Online Live Streaming (OLS) of Games}

Online live streaming (OLS) is like live playback of YouTube video clips. By live streaming audio-visual (AV) data, the service enables users of web cameras or screen capturing programs to send their AV files to viewers all over the world. Given today's ever-increasing bandwidth of mobile networks, OLS services can also be offered with simply a smartphone. This offers easy access to a multitude of real-time broadcasts rather than simply pre-recorded files [10]. In addition, the viewers of a live stream may know who they are watching with and interact with them or even the broadcaster (the host of the live show on that same channel) through a dialog box. This feature contributes greatly to the interaction and social networking among the viewers of an OLS show.

Viewing a live broadcast on the Internet has become an important online activity in Taiwan, with mobile devices having become a major platform, according to a report titled "A Survey on Viewing of Online Live Broadcasts in Taiwan" published by the Institute for Information Industry [11]. In addition, Facebook's promotion and improvement of its OLS services these years also shows that live streaming will remain a trend as an online activity in the future.

In addition, we also referred to the Institute for Information Industry's survey and the data of television broadcasts. The results are shown in Table 1, which reveals that today's Internet users are all content creators. They do not passively wait for what will be televised. This phenomenon is quite different from what it used to be like in the past, with traditional TV networks becoming less influential in the market due to OLS services. The biggest difference between the two categories, however, lies in the social interaction and the proactivity/passivity of content.
The viewers of an OLS show become a part of it as well as content providers even if there is the broadcaster hosting the show. It is this unpredictability that makes live streaming interesting. For advertisers, it is easier to measure the viewership of an OLS show, take advantage of its word-of-mouth effect on production promotion and invest smaller amounts of money when compared with traditional televised programs, whose viewership is harder to measure and whose profitability depends more on TV ratings.

\begin{tabular}{|c|c|c|}
\hline Category & $\begin{array}{l}\text { Traditional TV Live } \\
\text { Shows }\end{array}$ & $\begin{array}{l}\text { Online Live Streaming } \\
\text { Shows }\end{array}$ \\
\hline Features & Unilateral viewing & Real-time interaction \\
\hline Viewership & Mostly aged 35 or older & Mostly aged 35 or younger \\
\hline $\begin{array}{l}\text { Content of } \\
\text { programs }\end{array}$ & $\begin{array}{l}\text { More } \\
\text { professional-generated } \\
\text { content (PGC); more } \\
\text { costly productions; } \\
\text { mostly ball games, } \\
\text { concerts and news } \\
\text { coverage }\end{array}$ & $\begin{array}{l}\text { More user-generated } \\
\text { content (UGC); less costly } \\
\text { productions; more gaming } \\
\text { events; shopping programs, } \\
\text { academic seminars and } \\
\text { shows featuring } \\
\text { good-looking online } \\
\text { sensations }\end{array}$ \\
\hline $\begin{array}{l}\text { Sources of } \\
\text { revenue }\end{array}$ & Advertising & $\begin{array}{l}\text { Advertising; selling virtual } \\
\text { items; profits shared by the } \\
\text { broadcaster and advertiser }\end{array}$ \\
\hline $\begin{array}{l}\text { Advertising } \\
\text { criteria }\end{array}$ & $\begin{array}{l}\text { Programs with more TV } \\
\text { ratings and exposure } \\
\text { favored by advertisers; } \\
\text { hard to measure the } \\
\text { viewership due to poorer } \\
\text { interactivity }\end{array}$ & $\begin{array}{l}\text { Staying on top of the } \\
\text { number of current viewers } \\
\text { and user profiles; easier to } \\
\text { choose what to advertise; } \\
\text { more proper for small } \\
\text { investments and partnership } \\
\text { advertising projects }\end{array}$ \\
\hline
\end{tabular}

$\overline{\text { Source: Institute for Information Industry, 2015; Wang and Chen (authors of }}$ this study)

\section{Video Calling/Conferencing Platform}

As discussed above, TV networks do not favor less popular sporting events because broadcasting equipment can be expensive and games with a narrow viewership are not lucrative. Even if such games are aired on TV, they are mostly pre-recorded rather than being broadcast live. However, today's increasingly mature streaming technology prompted us to consider whether it is possible to live broadcast games on the Internet. Most OLS platforms offer only one camera angle (observation as of February 2017). This means that only a single web camera is used to capture one camera angle for live broadcasts. That being said, sports games (particularly ball games) often require multiple camera angles, so typical OLS platforms would not be useful for sports broadcasting. Although it is possible for us to design a piece of software tailored for our research, we consider this risky and against the latest concept of 'running lean' [12]. This is because we still know little about what might be a problem and what users need exactly. Therefore, we used many of today's video calling/conferencing services in combination with OLS platforms to live broadcast sports games.

Video calling/conferencing is an application of streaming technology. It is multicast simultaneous transmission of AV data. Fig. 1 demonstrates the structure of a video 
calling/conferencing system, according to Lee [13]. As part of the structure, a camera, a keyboard, a display, a microphone and an earphone on one local end are connected to a real-time video transmission system. The system is then linked to the devices on the other local ends by using a network adaptor. Naturally the real-time video transmission system is the major part of the structure. AV data is captured using a grabber card, stored into a buffer zone and encoded according to a certain encoding format. To make transmission more efficient, the data is usually compressed and segmented into packets. Inside the packet is information on types of compression encoding, timestamps, and serial numbers of packets. These steps are done to form a header. Packets with a body and a header are sent to the Internet using a network interface card (NIC). After a packet is received on the remote end, the system will decompress it by confirming whether its content and timestamp are accurate in accordance with its header. Decompressed packets are displayed via different devices [14].

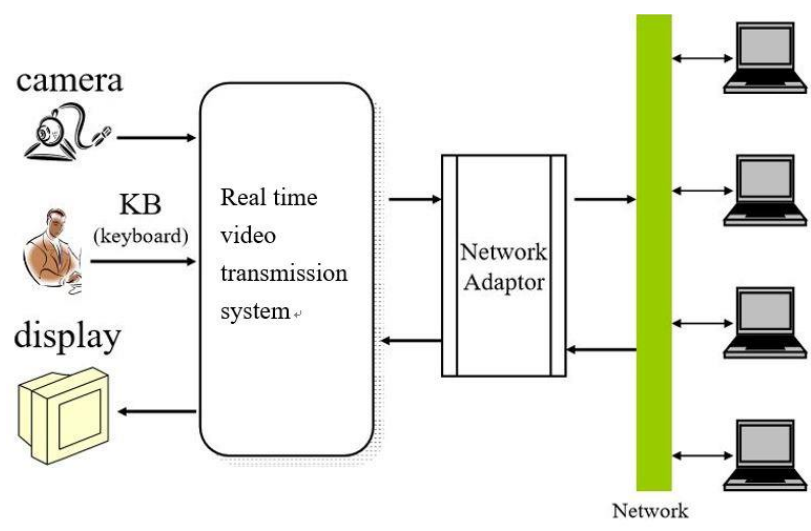

Fig. 1. Video calling/conferencing system. Source: Lee, 2005.

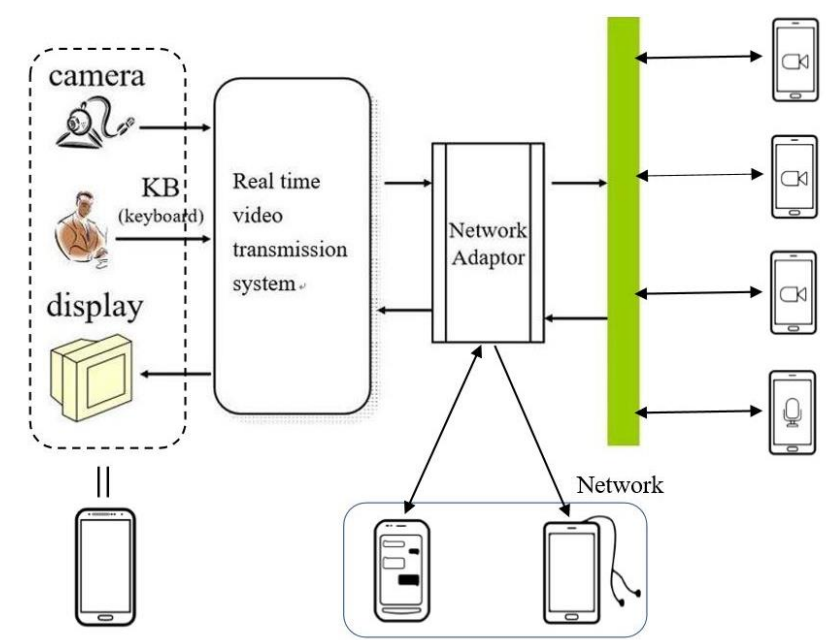

Fig. 2. Broadcasting a game via a video calling/conferencing platform Source: Wang and Chen (authors of this study).

There have been a variety of protocols during the process of technical development. The focus of our research, however, is not on these technical issues. We attempted to explore whether the current video calling/conferencing technology can be applied to broadcasting. Fig. 2 is the result of expanding the structure in Fig. 1, where the camera, keyboard and display are replaced by simply a smartphone. The smartphones on the right side of Fig. 2 - which are used to film or record voices - work in the same way as those on the left side. As for the two smartphones in the bottom of Fig 2 , we can see them as either a set of devices or separate ones, with the right one used to capture $\mathrm{AV}$ data from the spectators of a game and the left one to offer a platform, where spectators can interact with each other and share ideas about the game (as discussed above in the literature review section).

The system in Fig. 2 can either work as a whole or be considered the combination of a video calling/conferencing platform and a piece of broadcasting equipment. As we experimented with prototype testing for our research, we used Google Hangouts to live broadcast on YouTube. The following methodology section will describe how we conducted this test.

\section{DATA COLLECTION AND ANALYSIS}

\section{A. Broadcasting Team}

Sportscasting (the reporting of a sports event) calls for teamwork, with at least a camera crew, an anchor, and a commentator/analyst. While filming a sporting event, the camera crew needs to capture the images of players from different angles in order to keep the spectators on top of game plays. As per the definition in the study of journalism, the broadcast of a ball game usually consists of two parts: story and discourse. The former is about narration, the latter about interpretation or commentary from the speaker's own perspective [15]. Liao mentioned that there are 11 categories in what an anchor talks about a game: description, explanation, compliment/criticism, speculation, comparison, retelling, reflection/review, metaphor/simile, suggestion, belief and doubt [16]. In the past, when there were only radio broadcasts, faithful description played a vital part because fans relied heavily on the anchor of a game talking about it. With television broadcasts now ubiquitous, viewers see images. This relieved the anchor of his/her burden of talking about it, but he/she had to comment more on it. To make sportscasting less boring, some broadcasters started to include an analyst working together with an anchor on the reporting of a game.

To bring our prototype testing closer to real-world television broadcasting, we formed a six-person team. Three of them used smartphones to capture live stream screens, with two acting as the anchor and analyst, and the other one switching between screens.

\section{B. Prototype Testing Game}

To avoid potential disputes over broadcasting rights, we chose untelevised games for prototype testing via smartphones. As Google Hangouts does not support the zoom-in/zoom-out function, we had to choose a sport that makes it easier for us to capture images using a smartphone.

We then select a first-round game played by Group B teams in the HBL in 2016. Figs. 3-5 show three screenshots, which were taken from three camera angles. The right bottom of each screenshot shows that five smartphone users were conducting a videoconference, with three of them filming the game, one switching the screens and the other broadcasting the voice of the anchor and the commentator. 


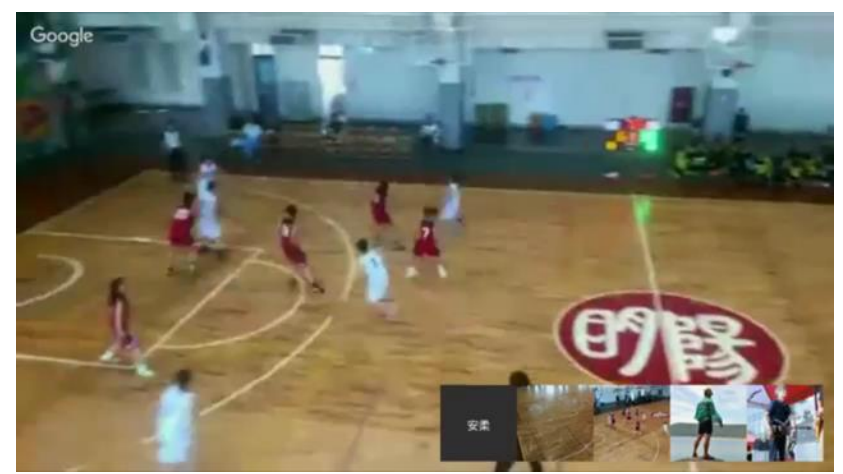

Fig. 3. Camera angle \#1.

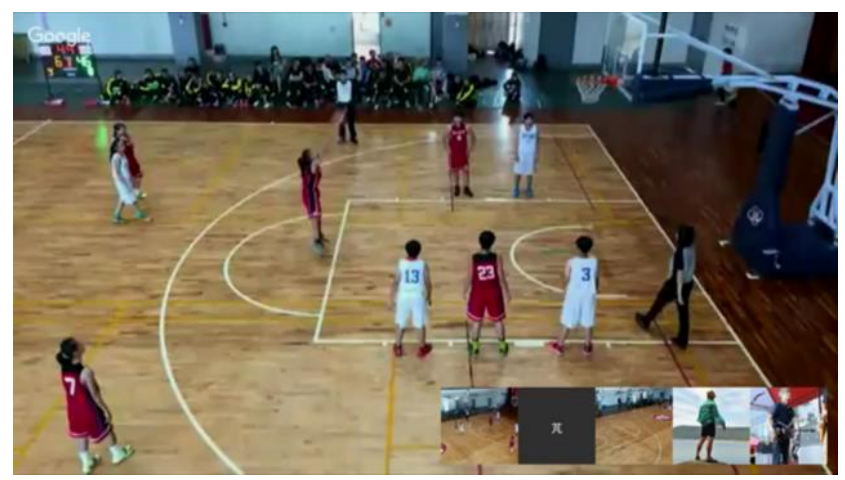

Fig. 4. Camera angle \#2.

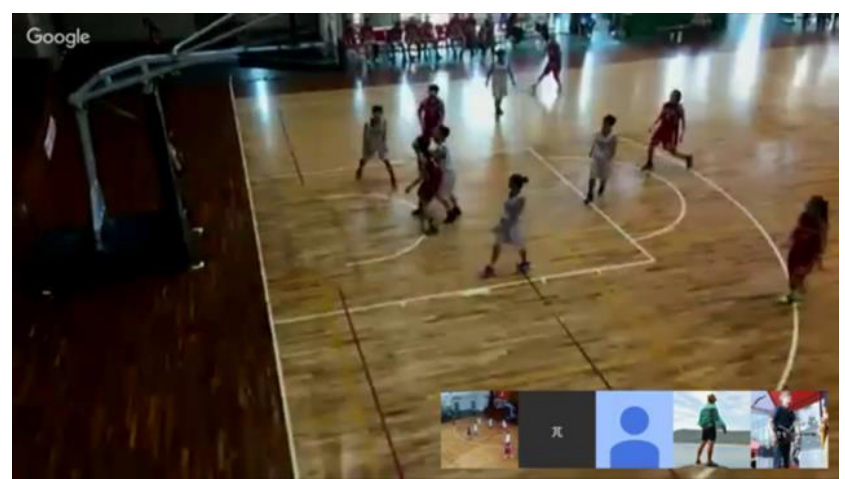

Fig. 5. Camera angle \#3.

\section{Post-game Questionnaire Survey}

Our model for prototype testing was designed to explore the feasibility of smartphone-based broadcasting and the spectators' feeling and user experience. To this end, we asked a group of viewers to watch the live broadcast of the game using a big screen in a classroom for a period of one hour. The same test was done on another day on a different group of viewers watching another game.

As part of the questionnaire survey, the viewers of the first game were asked to write down three advantages and three disadvantages about the live broadcast. A total of 28 questionnaires were then collected, encoded and analyzed statistically. The top three advantages were: 'attractive reporting style,' 'better engagement in sports' and 'more chance to watch games of various kinds;' the top three disadvantages were: 'image quality not clear enough,' 'slightly unstable movements of camera' and 'insufficient camera angles.' Figs. 6-7 show the statistics of advantages and disadvantages of the live broadcasts.

We redesigned our survey in accordance with the feedback from the first group of viewers. The revised questionnaire contained four close-ended questions about their comments on: (1) the anchor and analyst; (2) the live streaming; (3) the game itself; and (4) the whole broadcasting model. Respondents had close-ended five-eight options for each question and were able to choose more than one option.

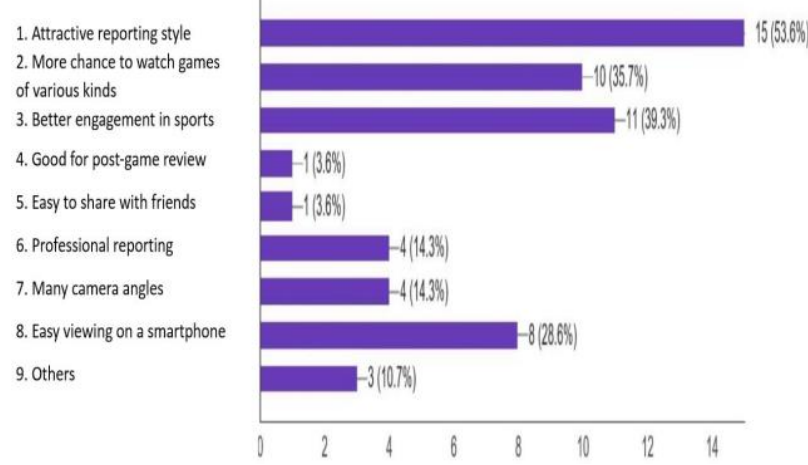

Fig. 6. Statistics of advantages of the live broadcasts.

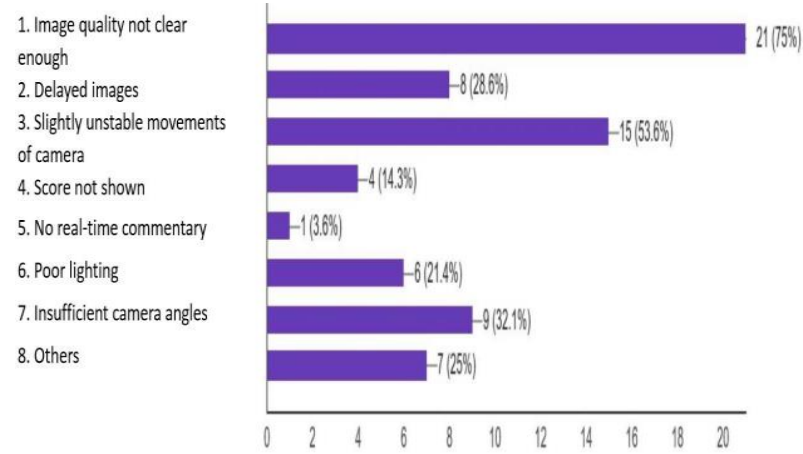

Fig. 7. Statistics of disadvantages of the live broadcasts.

A total of 21 questionnaires were collected. The results show that: (1) Having a sportscaster reporting the game attracted more spectators; (2) Poor lighting and shaky camera movements undermined the broadcasting quality; (3) Viewers/spectators would find a game more engaging if it is played by any of their friends; and (4) 'Real-time' broadcasting is the core value of user experience. Figs. 8-11 show the statistics of the four questions.

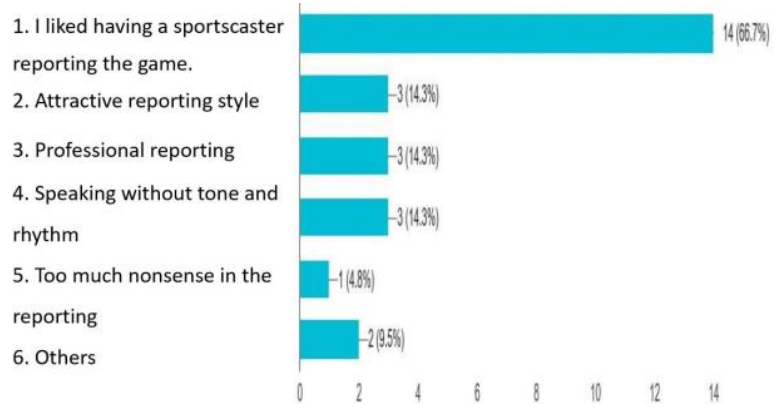

Fig. 8. Statistics of comments on the anchor and analyst.

1. Acceptable image quality
2. Poor image quality
3. Shaky camera movements
4. Many camera angles
5. Insufficient camera angles
6. Poor lighting
7. The score should be
displayed in the bottom of
the screen
8. Others

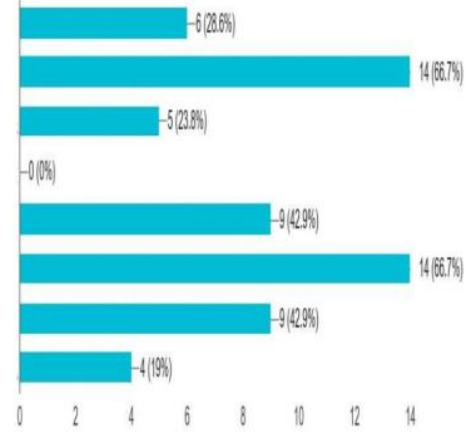

Fig. 9. Statistics of comments on the live streaming. 


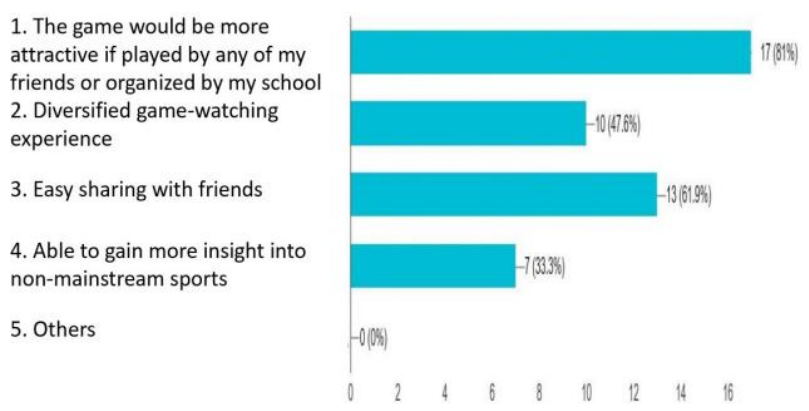

Fig. 10. Statistics of comments on the game itself.

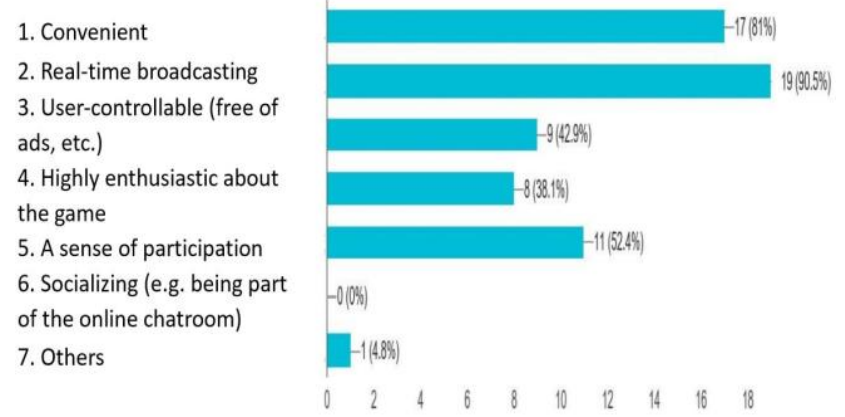

Fig. 11. Statistics of comments on the whole broadcasting model.

\section{Discussion, CONCLUSION AND SUGGESTIONS}

\section{A. Discussion}

1) Analysis of the first questionnaire survey

As far as the advantages of the prototype testing are concerned, most respondents lauded the 'reporting style,' 'diversified game-watching experience', 'convenience' and better engagement in sports. The factors 'professional reporting' and 'many camera angles' were ranked in the middle. In addition, the factors 'good for post-game review' and 'easy to share with friends' were not influential as advantages probably because the respondents were not players of the game or their friends.

In terms of disadvantages, 'image quality not clear enough' and 'shaky camera movements' were commonly considered defects. It is interesting to note many of the respondents saw 'insufficient camera angles' as a disadvantage. The factor 'many camera angles' was ranked in the middle. This was probably because Google Hangouts does not support zoom-in and zoom-out. As a result, the viewers were not able to see close-up shots of plays from various angles. This reduced the effectiveness of the videoconferencing software greatly. Moreover, technical problems related to the hardware and network environment were responsible for 'delayed images' and 'image quality not clear enough,' which was beyond control as we were still in the stage of prototype testing. 'Score not shown' was seen as a defect by many respondents, with 'no real-time commentary' by only a few. This observation was in line with 'reporting style' outscoring 'professional reporting' as an advantage.

2) Analysis of the second questionnaire survey

While most respondents considered it necessary to have an anchor/analysist, the professionalism of the anchor/analysist and the reporting style were considered equally important. These two factors, however, were ranked in the middle.
In terms of the comments on the live streaming, two main factors were 'poor image quality' and 'poor lighting.' The former of the two was attributable to the poor reception inside the basketball court, which lowered the uploading speed of the smartphones. The latter was because that the court manager did not expect many spectators to come and then turned off the main light for environmental concerns. 'Score not shown' and 'insufficient camera angles' were due to software limitations and the absence of zoom-in/zoom-out function, respectively, which greatly reduced the effectiveness of Google Hangouts as a videoconferencing platform. As to 'shaky camera movements,' we substantially improved this problem after receiving feedback on the first live stream. The factor 'poor image quality' was acceptable as many of our subjects realized it was a smartphone-based live broadcast.

Regarding the game itself, many respondents thought that the game would have been more attractive if played by any of their friends. They also hoped that such a model could help increase the visibility and exposure of athletes. In addition, 'diversified game-watching experience' was also among the benefits that a smartphone-based live broadcast can offer.

Lastly, the respondents considered 'convenient' and 'real-time broadcasting' the core values that lie in the whole broadcasting model. This has highlighted the importance of a smartphone-based live broadcast. In addition, there was also 'a sense of participation' that engaged more people to play a sport, thereby gaining more of their attention and enthusiasm. These were all considered important factors. 'Socializing' was deemed extremely unimportant probably because that the viewers watched the live broadcast for a period of only ten minutes while staying concentrated fully on the new broadcasting model, which might have shifted their attention from socializing or interacting with others.

\section{B. Conclusion and Suggestion}

We have concluded and listed several suggestions as follows based on our prototype testing.

1) It is feasible to live broadcast a game on a smartphone. The broadcasting model will be more well-established if complete with the zoom-in/zoom-out function and display of the game's score on a video calling/conferencing platform.

2) Network bandwidth was mainly responsible for the unclear image quality of the uploaded film. It would have helped if better lighting (i.e. turning on the main light in our test) was offered on the court. As to the shaky camera movements, the camera holders had to hold their smartphones more firmly.

3) Our broadcasting model was generally recognized. In particular the individual reporting style of the inexperienced anchor intrigued many of the viewers. The convenience and real-time broadcasting of the model were also important in increasing the exposure of athletes and engaging more people to play a sport.

4) The socializing feature of Internet-based broadcasting in some literature was not tested in our prototype model. We suggest that future researchers focus more on this issue.

To sum up, this study discovered a new way to broadcast 
sports games. It is believed that this model can become more mainstream in the near future with technological advances in software and hardware.

\section{REFERENCES}

[1] C. P. Shih, "The study of the interactive relationship between the media and sport commercialization," Physical Education Journal, no. 28, pp. 87-100, March 2000.

[2] H. Z. Li, "Effect of traditional mass media on sports development," The University Physical Education \& Sports, no. 52, pp. 89-94, February 2001.

[3] L. A. Wenner, "Media, sports, and society: The research agenda," Media, Sports, \& Society, $1^{\text {st }}$ ed., London: SAGE, 1989, pp. 13-48.

[4] C. J. Ni, "CTSSF to add three more HBL live broadcasts of women's basketball games to solve disputes," November 23, 2015.

[5] G. J. Conklin, G. S. Greenbaum, K. O. Lillevold, A. F. Lippman, and Y. A. Reznik, "Video coding for streaming media delivery on the internet," IEEE Transactions on Circuits and Systems for Video Technology, vol. 11, no. 3, March 2001, pp. 269-281.

[6] M. Chesire, A. Wolman, G. M. Voelker, and H. M. Levy, "Measurement and analysis of a streaming-media workload," in Proc. the 3rd USENIX Symposium on Internet Technologies and Systems, March 26-28, 2001, pp. 1-12.

[7] A. D. Gelman, S. Halfin, and W. Willinger, "On buffer requirements for store-and-forward video on demand service circuits," IEEE Global Telecommunications Conference, GLOBECOM '91. 'Countdown to the New Millennium. Featuring a Mini-Theme, 2-5 December, 1991.

[8] V. N. Padmanabhan, H. J. Wang, P. A. Chou, and K. Sripanidkulchai, "Distributing streaming media content using cooperative networking," in Proc. the 12th International Workshop on Network and Operating Systems Support for Digital Audio and Video, 12-14 May, 2002, pp. 177-186.

[9] H. Deshpande, M. Bawa, and H. Garcia-Molina, "Streaming live media over a peer-to-peer network," Stanford Database Group Technical Report, 2001.

[10] B. Brouwer, "What live Streaming means for content publishers," EContent, vol. 38, no. 8, 2015, p. 11.

[11] Y. C. Weng, "A survey on viewing of online live broadcasts in Taiwan," Foreseeing Innovative New Digiservices, Institute for Information Industry, October 15, 2015.
[12] A. Maurya, Running Lean, Sebastopol, California: O’Reilly Media, Inc. 2013.

[13] C. W. Lee, "The study and analysis of real-time video transmission in internet," Thesis for master of science, Dept. of Elect. Eng., National Cheng Kung University., Taiwan, Taiwan, 2005.

[14] I. S. Twu, "Design of real-time control protocol hardware for video conference system," Thesis for master of science, Dept. of Elect. Eng., National Cheng Kung University., Tainan, Taiwan, 2003.

[15] Y. Tsa and K. J. Tsang, "News as narrative: A theoretical analysis of news structure," Mass Communication Research, no. 58, pp. 1-28. 1999.

[16] K. W. Liao, "TV relay 2011 CPBL Taiwan series, play by play man and commentators commentary description of baseball content - The ELTA sport channel as example," Thesis for master, Dept. of Leisure Services Management, Chaoyang University of Technology., Taichung, Taiwan, 2014

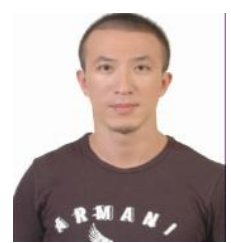

K. L. Wang works as an associate professor in the Department of Recreation and Leisure Industry Management, National Taiwan Sport University. His area of interest is the law and public policy issue in leisure, entertainment, and sport field. Because of his economics major background, he did many labor economics in sport industry such as the topics of coach effort on players performance, or the effect of good looking on the players' popularity...etc.. Recently, he did many studies about the image right, sport IT management, patent and intelligent property management, public policy and legislation regarding the sport industry based on the approach of economics of law. He also got the award in the competition of innovation and new business venture in Taiwan.

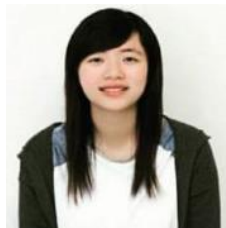

Y. H. Chen graduated from the master program of international sport affairs, National Taiwan Sport University. Her interested research field is about the sport management and sport communication. She works in the Market Development Division of the 2017 Taipei Universiade Organizing Committee now. 